\title{
Autonomous Medication Distributor Through Implementation of Bresenham's Line Algorithm
}

\author{
Brandon Duran, $\mathrm{BS}^{1}$, Diego Villegas, $\mathrm{BS}^{1}$, and Sebastian Valencia, $\mathrm{BS}^{1}$ \\ ${ }^{1}$ Vaughn College of Aeronautics and Technology, USA, brandon.duran@vaughn.edu, \\ diego.villegas@vaughn.edu,sebastianandres.valenciachango@vaughn.edu \\ Mentors: Dr. Hossein Rahemi, Dr. Mohammed Benalla, Dr. Miguel Bustamante, Dr. Amir Elzawawy \\ Mohammed.benalla@vaughn.edu, Miguel.bustamante@vaughn.edu, Amir.elzawawy@vaughn.edu, \\ Hossein.rahemi@vaughn.edu
}

\begin{abstract}
The goal of this project is to create an autonomous system that will dispense pills of various sizes and shapes. The overall objective is to utilize the invention to assist in dispersing the appropriate amount of medication for prescribed patients at designated times. It is imperative to take the proper amount of medicine at the specific times prescribed. In today's fast-paced world, it is easy to forget to keep track of simple tasks, such as taking the necessary medications needed for individuals with certain conditions. As people grow older, the ability to recall every day's tasks begins to deteriorate. The autonomous pharmaceutical dispenser will utilize a mechanism that consists of an $x, y$, and $z$-axis movement to maneuver a suction unit to transport pills from a capsule to a delivery location. Bresenham's line algorithm will be used to control the motors of the 3D axes movement systems. The dispenser will be able to store a month's supply of tablets with the ability to dispense up to twelve different types of medicines. The system will come with a wireless gadget that will receive notifications from the distributor, alerting the patient to take their medication. In case the user forgets to take his or her medication, the portable device will receive a message. Moreover, an excel spreadsheet will record a detailed summary of the administered medicine. The objective is to create a cost-effective and reliable alternative product for patients to use for their medical needs.
\end{abstract}

Keywords-- Autonomous, ESP32, algorithm, safety, efficient.

\section{INTRODUCTION}

The need for an automated pill dispenser for those individuals who tend to forget, or need to be reminded, to consume mandatory medication is apparent. Undoubtedly, consequences arise due to the inadequacy of remembering medication doses. Because of failure to comply with prescribed treatment, over 100,000 people are estimated to die each year in the United States [1]. There are alternative solutions to assist with helping patients to remember to take their medication. For example, there are downloadable apps to help people to take their prescribed medication. On the other hand, there are unnecessary hospital admissions for about half of patients who do not take medications as prescribed, costing the U.S. healthcare system an estimated \$290 billion a year [2]. As a result, doctors utilize a high-tech approach, such as a smartphone app, that sends patients reminders to take their medication at the designated time. The use of new and convenient technological options are appealing; however, there is still a lack of supporting data on which health providers can base their recommendations and the

Digital Object Identifier (DOI):

http://dx.doi.org/10.18687/LACCEI2020.1.1.651

ISBN: 978-958-52071-4-1 ISSN: 2414-6390 services they provide. Tech applications may help resolve the problem, but they do not completely address personal needs.

Pill dispensing systems suit a wide range of patients; however, several studies have reported less than optimal results with pilldispensing methods [3]. Many different potential systems are more efficient than predecessors, but they have not yet reached the objective. Many of these devices are problematic due to their unreliability [4]. Consequently, improving the efficiency of the apparatus and offering an advanced product to society is the idea behind innovating a medical dispenser. PharmAid is a contribution towards improvement of the dilemmas regarding autonomous medicine dispensers. This system presents a more reliable and trustworthy product; however, the team intends to improve the distribution mechanism, the compactness, and the practicality of PharmAid. During our careful analysis process, the team encountered a distinct approach towards enhancing the performance of the prototype.

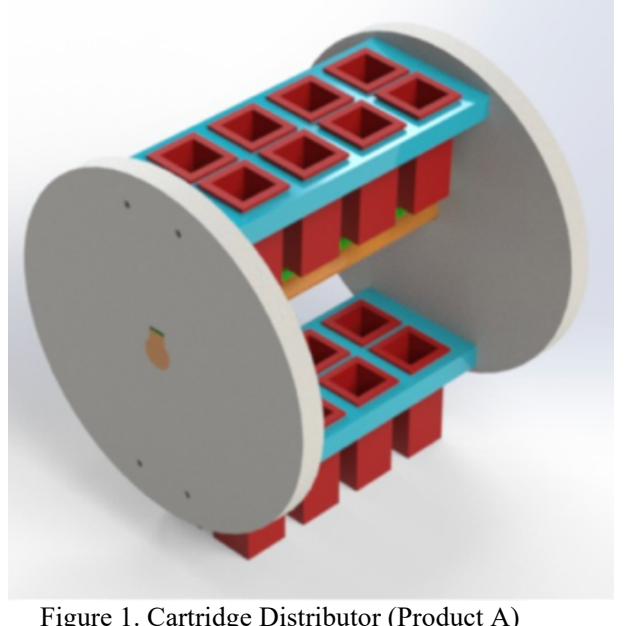

The previous concept of using stepper motors to rotate the cartridge distributor can be seen in Fig. 1. The mechanism provides up to sixteen different compartments that support between 20 to 90 different tablets depending on the tablet size. As ideal as this seems, the distributor can take too long in aligning the cartridges to the suction cup. Replacing the rotation of the rounds to an $\mathrm{x}, \mathrm{y}$, and $\mathrm{z}$ motion using Brenham's line algorithm reduces the distribution time. Throughout the research process documented in this paper, improvements will be made to the cartridge distributor. 


\section{ECONOMIC IMPACT}

The market for fully autonomous pill dispensers is still in its early stages. At the moment, PharmAid is the least expensive product on the market. Although it is cheaper, it will still compete with, and surpass, the leading fully autonomous pill dispenser, HERO. Similar products include: the MedaCube, Auto Pill Automatic Pill Dispenser, and Livi Smart Home Medication Dispenser. Although these pill dispensers offer a game changing solution, they come with a hefty price tag. The economical comparison can be seen below in table 1. For this prototype (PharmAid), a total of $\$ 574.17$ was spent. If this product were to go into manufacturing, this price could be reduced by $25-50 \%$. Therefore, we have estimated that this project can be sold for $\$ 999$. As shown below, there are similar products on the market at a higher cost.

Table 1. Economical Comparison

\begin{tabular}{|c|c|c|}
\hline & PharmAid & Hero \\
\hline Cost & $\begin{array}{c}\$ 799 \\
\text { Lease } \$ 24.99 \text { per } \\
\text { month/12 months } \\
\text { \$59.00 Initial fee }\end{array}$ & $\begin{array}{c}\text { *Cannot be } \\
\text { Purchase } \\
\text { Membership - } \\
\text { \$29.99 - \$99.99 } \\
\text { Initial Fee }\end{array}$ \\
\hline $\begin{array}{l}\text { Medication } \\
\text { Storage }\end{array}$ & $\begin{array}{l}12 \text { Medications } \\
\text { (30 to } 90 \text { pills each) }\end{array}$ & $\begin{array}{l}10 \text { Medications } \\
\text { (20 to } 90 \text { pills) }\end{array}$ \\
\hline $\begin{array}{l}\text { Security } \\
\text { System }\end{array}$ & Passcode + Key & Passcode + Key \\
\hline Notifications & $\begin{array}{c}\text { Phone }+ \text { On screen } \\
+ \text { Sound }+ \text { Lights }+ \\
\text { Buzzer }+ \text { Wearable } \\
\text { Gadget }\end{array}$ & $\begin{array}{c}\text { Lights + Sound + } \\
\text { Phone }\end{array}$ \\
\hline
\end{tabular}

\section{METHODOLOGY}

\section{A. Focus}

This innovation aims to make the dispense of medicine as autonomous as possible. For this research, several analyses using an X-Y-Z mechanism demonstrated improvements in the automated medicine dispenser. Different components, such as a $24 \mathrm{~V}$ vacuum compressor and a humidity sensor, were also determined to improve the system. The idea behind these analyses is to strengthen the concept of the autonomous medicine dispenser by making it quicker and more productive. The system needs to be quicker in the case of multiple medications needing to be taken at a specific time.

\section{B. Mechanism \& Programing}

The project uses a movement system similar to a 3D Printer. The vacuum tube end moves on the $\mathrm{x}-\mathrm{y}$ axis using two motors. The motors are connected and collaborating using shafts and GT2 pulleys to run the vacuum tube to the intended locations. Limit switches control the $x-y$ axis to operate within the frame of the design. The switches prevent the motors from putting force on the frame. For the $\mathrm{z}$ axis mechanism, a tray holds cartridges filled with medicine and is mounted to a lead screw. The plate moves up and down on the lead screw, which is driven by a coupled motor. The mechanism uses a vacuum pump with a nozzle at the end of a connecting tube in order to grasp one pill at a time, regardless of the size or shape of the given set of tablets. Bresenham's line algorithm takes a notable impact on the device and the code. The cartridge distributor shown in Fig. 1 is an idea made to be efficient; however, the $x, y$, and $z$ motions allow the machine to improve the fast response of the unit, while being practical at the same time. Bresenham's algorithm for plotting a two-dimensional line segment is elegant and efficient in its deployment of mid-point comparison and integer arithmetic. [5] The central unit works with a twodimensional motion to control the suction system. Meanwhile, the code follows the concept of the algorithm, as do most CNC machines or 3D printers. The model is shown in Fig. 2. The medical dispenser microcontroller uses an Arduino ide. The code operates the X-Y-Z axis, laser distance sensor, stepper motors, pressure sensor, temperature sensor, and LCD. The product has simple refillable containers in a tray; the suction system transports the necessary dosage of medicine from the boxes to the deposit area at the designated time. The microcontroller receives information on how far to move the zaxis up to the vacuum pump through a laser distance sensor to retrieve the doses. The vacuum pump can identify when it has latched onto a pill with the aid of a pressure sensor. The sensors, vacuum pump, and microcontroller allow the medicines to be transported from the various containers to the deposit location.

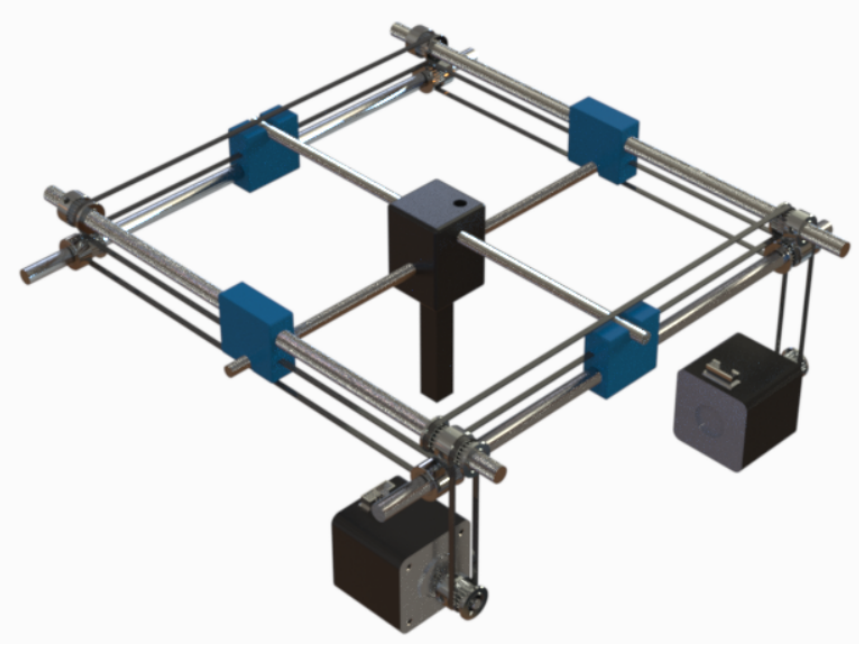

Figure 2. X-Y Axis Movement Mechanism (Product B)

Fig. 2 shows the model that uses Bresenham's algorithm. The $\mathrm{z}$ axis mechanism lifts the tray that holds the cartridges, aligning them to the right position of the suction system. As seen in Fig. 3 , the stepper motor links to the lead screw using a coupler, raising and lowering the plate. All medicine will be stored in cartridges as shown in Fig. 4. 


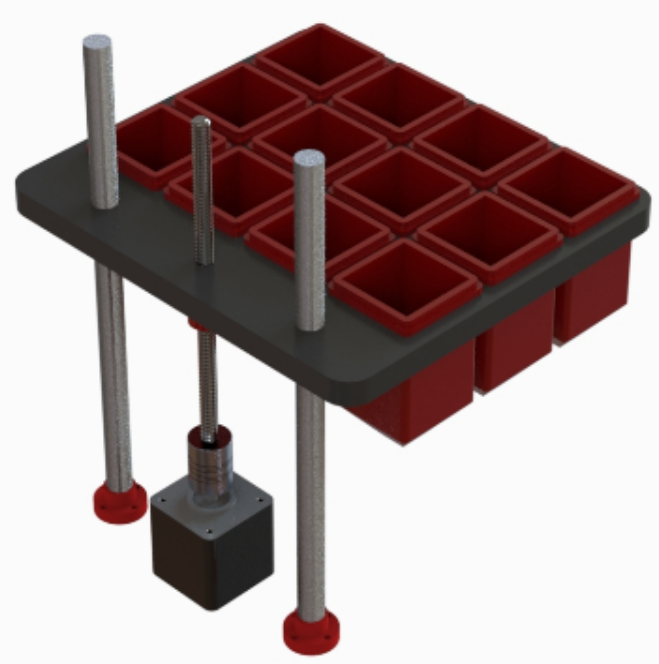

Figure 3. Z Axis Movement Mechanism (Product B)

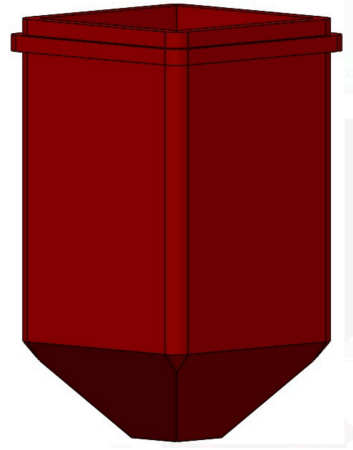

Figure 4. Pill Cartridge

\section{Bresenham's Line Algorithm}

This algorithm follows basic arithmetic concepts, where the slope of the line is unknown [6]. Equation (1) provides the formulation to find the slope of the line. In this formula, "m" represents the slope, $\left(x_{2}, y_{1}\right)$ is the coordinate of the first point of the line, and $\left(x_{2}, y_{2}\right)$ is the coordinate of the second point of the line. The two coordinates can be represented by $\mathrm{dx}$ and $\mathrm{dy}$.

$m=\left(y_{2}-y_{1}\right) /\left(x_{2}-x_{1}\right) \equiv d y / d x$.

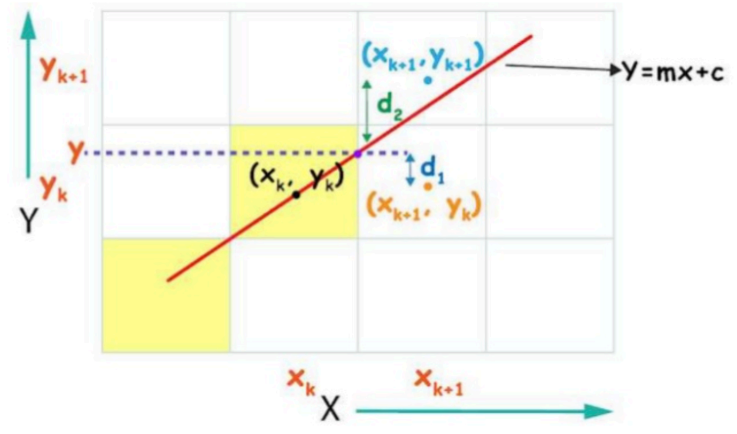

Figure 5. Position Coordinate
The points where the suction tube is going to pass are measured. Sometimes, the system passes through two different locations at the same time; then, the device has to choose one of the points to go. Bresenham's algorithm calculates the distance from the intersection points and selects the smaller one. The equation of the line (2) represented by ' $y$ ' (a point in the y-axis), ' $x$ ' (a point in the $\mathrm{x}$-axis), ' $m$ ' (the slope of the line), and ' $b$ ' (y-intercept of the line).

$y=m x+b$.

The position coordinate provides a visual representation of Bresenham's line Algorithm. Through calculations, the slope of the line is determined. If the slope of the line is less than one, then ' $x$ ' will always be incremented. The first distance (3) between intersection point ' $y$ ' to the position ' $y_{k}$ ' is calculated. The second distance (4) with the upper section ' $y_{k+1}$ ' and intersection point ' $y$ ' is calculated.

$d_{1}=y-y_{k}$.

$d_{2}=y_{k+1}-y$.

Since $\mathrm{x}$ is always incrementing, the variable can be expressed as ' $x_{k}+1$ ' and ' $y_{k+1}$ ' as ' $y_{k}+1$ '. The first and second distance subtraction (5) applying the slope of the line (2) provides the general expression.

$d_{1}-d_{2}=2(d y / d x) *\left(x_{k}+1\right)-2 x_{k}+2 b-1$.

The distance difference (5) is multiplied by ' $d x$ ' in both sides to obtain decision parameter (6) ' $P_{k}$ ' and the constant value (7) ' $b$ '. The decision parameter and the constant value contributes with a smoother expression (8).

$P_{k}=d x\left(d_{1}-d_{2}\right)$.

$b=2 d y+2 d x(b)-d x$.

$P_{k}=2 d y\left(x_{k}\right)-2 d x\left(y_{k}\right)+b$.

The next parameter is the decision parameter upper level applying ' $P_{k+1}$ ' to the lower lever decision parameter (8). Combining the two expressions the change in decision parameter (9) is found.

$P_{k+1}=P_{k}+2 d y-2 d x\left(y_{k+1}-y_{k}\right)$.

The following boundary conditions are being considered:

- $P_{k}<0 \therefore y_{k+1}=y_{k}$

$P_{k+1}=P_{k}+2 d y$.

- $\quad P_{k}>0 \therefore y_{k+1}=y_{k}+1$

$P_{k+1}=P_{k}+2 d y-2 d x$.

For the initial decision parameter (12), the decision parameter (8) and the equation of the line (2) are being used.

$P_{o}=2 d y-d x$.

Equation (12) initial decision parameter is used when $m<1$. On the other hand, if $m>1$, the initial parameter (13) is going to change. The derivations shown above are the same, with the only exception the slope of the line (1). 
$P_{o}=2 d x-d y$.

\section{Power Supply}

A power converter converts $\mathrm{AC}$ power to $\mathrm{DC}$ power to supply the microcontroller, vacuum pump, and motors with power. The switch power supply is lightweight, highly efficient, and has a wide voltage range, making it the perfect fit for this project. Also, the small volume makes the unit compact. Switching the power supply's wide range of voltages allows the input of the appropriate energy needed for the mechanism [7]. The switch power supply has a similar characteristic to a transformer where it has a step up or down voltage so that it can maintain the appropriate ratio of the $\mathrm{AC}$ voltage to the converted $\mathrm{DC}$ voltage needed [8]. The power converter aligns with the standards of the project.

\section{E. Wearable Gadget}

In order to achieve the goal of precise medication management, the team incorporated a portable wearable gadget. A wearable gadget provides a reminder to users to take their medication, even when they are away from home or the pill dispenser. Although a phone app and website are part of future implementations, the team decided that this gadget could be used by anyone, regardless of age or level of technological experience. Using an ESP32 Wi-Fi/Bluetooth module, the team was able to create a master and slave relationship from the main unit to the wearable gadget. The portable device allows the patient to be reminded of the need to take medication at any given time away from the main unit. This will be accomplished by syncing the schedule for pill dispensing from the main unit (master) into the gadget (slave). Once it is time to take the medication, the gadget will beep, vibrate, and flash an LED. The ESP32 (Slave) schematics are shown in Fig. 7. A vibrating motor, LED, buzzer, one-hundred-ohm resistor, and a coin cell holder are connected to the ESP32 (Slave).

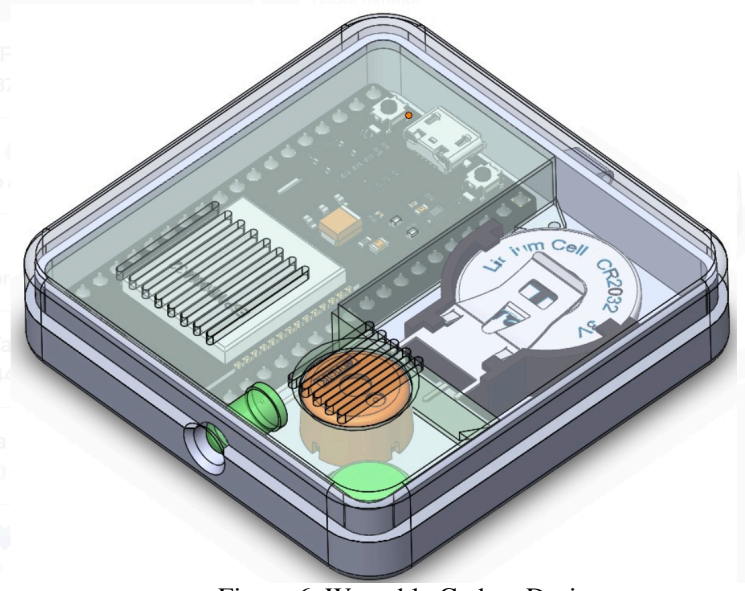

Figure 6. Wearable Gadget Design

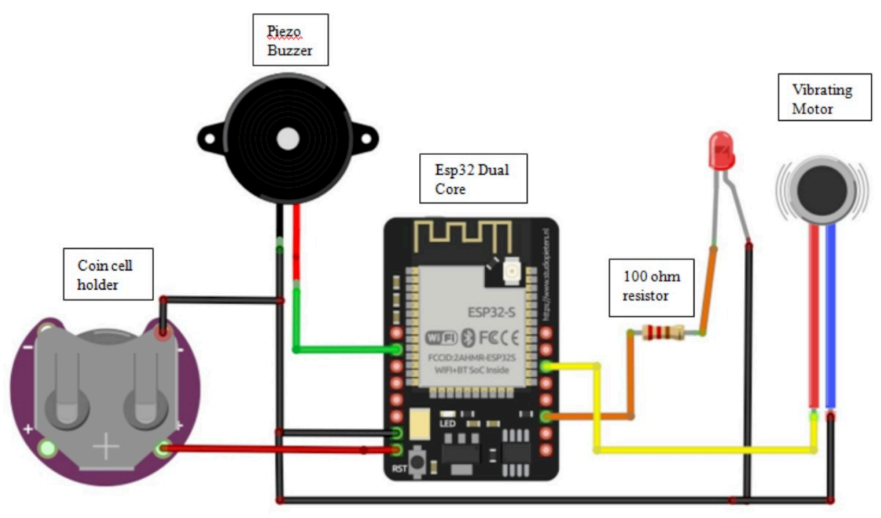

Figure 7. ESP32 (Slave) Schematics

The module emits a pulse reminding one that the pill is ready to be consumed. The module works without wi-fi, requires pairing, and has a peer-to-peer connection. Table 1 provides a table with the different properties of the module used in the master and slave connection. The implementation of this module into the project permits the creation of multiple gadgets that can be used for patient and care providers. For future analysis, the ESP32 can be modified to also send and receive signals.

\begin{tabular}{|c|c|}
\hline \multicolumn{2}{|c|}{ Table 2. ESP32 Properties } \\
\hline $\begin{array}{c}\text { Feature } \\
\text { Timescale (Physical, } \\
\text { Interpretation } \\
\text { Delay) }\end{array}$ & $0.1 \sim 1 \mathrm{~s}$ \\
\hline Storage & 520 KB SRAM \\
\hline $\begin{array}{c}\text { Interfaces/ } \\
\text { Resolution }\end{array}$ & $\begin{array}{c}\text { 12-bit ADC }(0-1.1 \mathrm{~V}), 8 \text {-bit DAC } \\
(0-3.3 \mathrm{~V}), \text { UART TTL (serial) }\end{array}$ \\
\hline Network & WLAN/Bluetooth \\
\hline Time Lagging & $\begin{array}{c}\text { RTC (internal real time clock) } \\
+\mathrm{NTP} \text { (network time protocol) }\end{array}$ \\
\hline Software & Arduino IDE \\
\hline Power & 5 Vdc, 3.3 Vdc \\
\hline
\end{tabular}

\section{F. Electrical Engineering}

The three stepper motors that guide the $\mathrm{X}-\mathrm{Y}-\mathrm{Z}$ axis are connected to a shield, which is connected to an Arduino Mega as shown below. In addition, five limit switches are connected to the same shield to control the axes movements and send signals to the Arduino. On the other hand, an RA8875 driver is used to connect the LCD to the Arduino Mega. A distance sensor, temperature sensor, humidity sensor, ESP32 (Master), and a pressure sensor are connected to the Arduino Mega as shown in Fig. 9. The distance sensor allows the motors to keep working until the sensor detects a pill. Also, the temperature sensor sends the output to the LCD where the patient can have accessibility to the data. In addition, the humidity sensor sends the output to the microcontroller, where it determines whether the fan turns on or off. The $24 \mathrm{~V}$ vacuum pump has enough power to pick up one pill at a time. In product $\mathrm{A}$, a $12 \mathrm{~V}$ vacuum pump was used, but the process was not quick enough. 


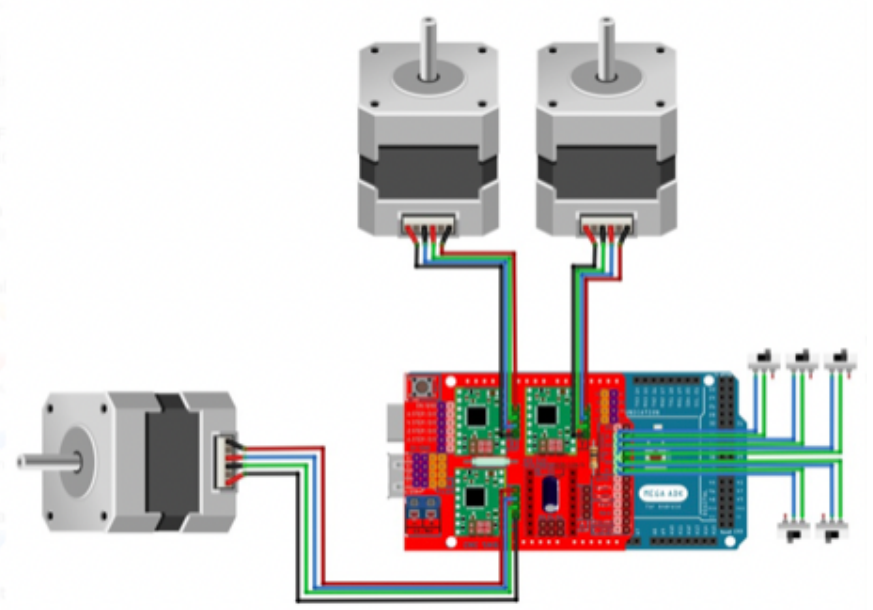

Figure 8. Motors Schematics

The ESP32 (master) connection to the microcontroller creates the connection directly to the slave. The connection allows the microcontroller to send signals when the medicine is ready. The signal received from the master sends a pulse to the slave and starts the vibration mode. The vibration mode lets the user know that it's time to take the medication.

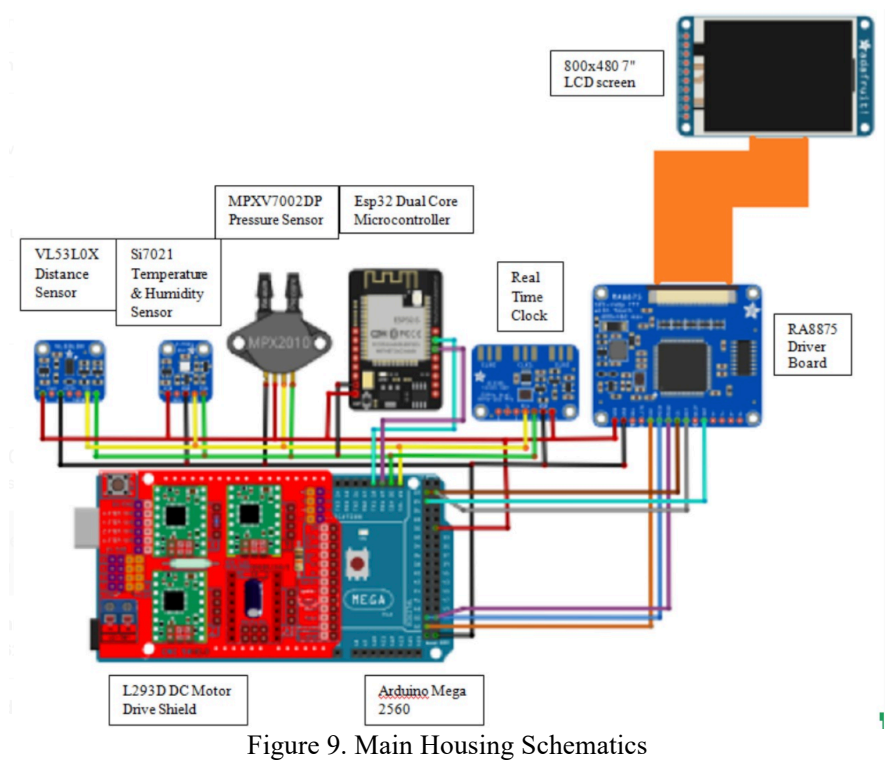

G. Safety Standards

For this project, different types of standards were taken into consideration, due to the overlapping genres of the project. The American Society of Health-System Pharmacist (ASHP) has regulations that the project followed. The most relevant standard is safety; therefore, a passcode grants the user access to the medicine. Also, a key-locking door, as shown in Fig. 10, allows the patient to refill the cartridges [9]. Additional committees such as the American National Standards Institute (ANSI) demand a close regulation for pharmaceutical concepts. The project proceeded to incorporate extra features to meet the requirements. The standards followed on the medical dispenser estimate a possible practical use in the industry [10]. The International Organization of Standardization (ISO) rules provided additional information for adaptation in the completion of the project[11].

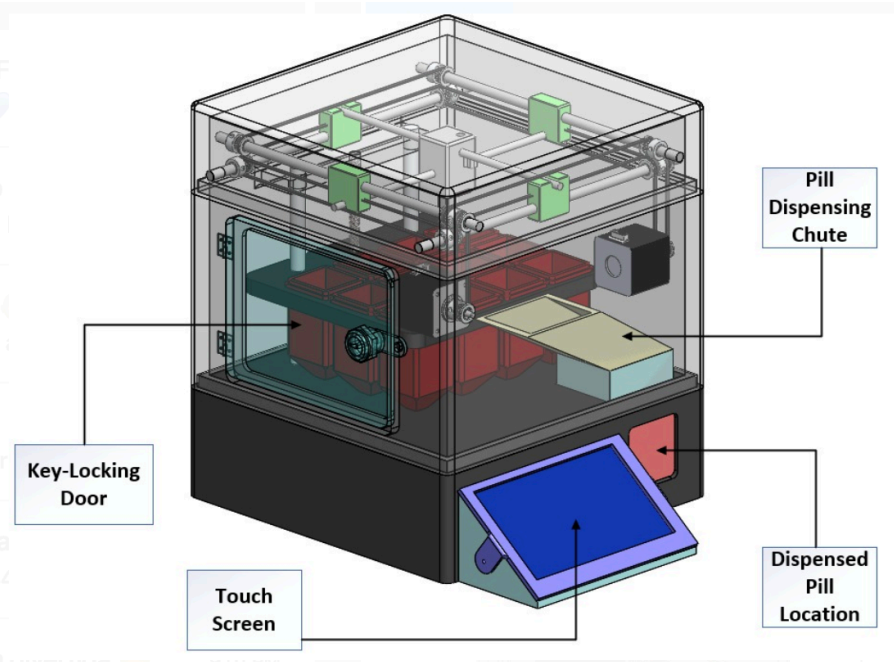

Figure 10. Main Housing \& Locking Mechanism

\section{ANALYSIS AND RESULTS}

Many elderly individuals take different kinds of medications daily. Keeping a record of the time, drug, and dosages can become a challenge for senior citizens. Throughout the research, the group identified the top nine medications most commonly used by the elderly, as indicated below. As shown in table 3, hydrocodone and metformin are the medications with heaviest usage, and levothyroxine one with the lightest. The investigation takes into consideration the weights of common pills to calculate the suction force needed to pick up each pill and prevent failure. For this analysis, a factor of safety of 2 is utilized, due to the properties of the medicine. A friction coefficient of 0.6 is used, due to the rough surface of the medicine. For this case, the suction cup and the direction of the force are horizontal, considering that the acceleration of the $\mathrm{Z}$-axis is $0.4 \mathrm{~m} / \mathrm{s}^{2}$. Considering the motion of the system and the known properties of the medicine, the theoretical holding force (14) represented by ' $F_{T H}$ ' ' $m$ ' (the mass), ' $g$ ' (gravity), ' $a$ ' (acceleration), ' $\mu$ '(coefficient of friction), and ' $S$ ' (factor of safety) and the suction force (15) represented by ' $F_{S}$ ' and ' $n$ ' number of suction pills can be determined using equations below.

\begin{tabular}{|c|c|}
\hline \multicolumn{2}{|c|}{$\begin{array}{l}\text { Most Prescribed Medication Weight } \\
\text { (mg) }\end{array}$} \\
\hline Hydrocodone & $300-1000$ \\
\hline Simvastatin & $5-80$ \\
\hline Lisinopril & $2.5-20$ \\
\hline Levothyroxine & $0.0025-0.3$ \\
\hline Amlodipine & $2.5-10$ \\
\hline Omeprazole & 20 \\
\hline Azithromycin & $250-500$ \\
\hline Metformin & $500-1000$ \\
\hline Hydrochlorothiazide & 25 \\
\hline
\end{tabular}


$F_{T H}=m(g+a / \mu) S$.

$F_{S}=F_{T H} / n$.

The suction force considers 30 pills per cartridge. The theoretical force and suction force results of each medicine can be seen in table 4 .

Table 3. Theoretical and Suctional Force

\begin{tabular}{|c|c|c|}
\hline Medicine & Theoretical Force $(\mathrm{N})$ & Suction Force $(\mathrm{N})$ \\
\hline Hydrocodone & 0.0197 & 0.00065 \\
\hline Simvastatin & 0.00158 & $5.267 \mathrm{E}-05$ \\
\hline Lisinopril & 0.00039 & $1.316 \mathrm{E}-05$ \\
\hline Levothyroxine & 0.000005 & $1.975 \mathrm{E}-07$ \\
\hline Amlodipine & 0.00019 & $6.584 \mathrm{E}-06$ \\
\hline Omeprazole & 0.00039 & $1.316 \mathrm{E}-05$ \\
\hline Azithromycin & 0.00987 & 0.00033 \\
\hline Metformin & 0.01975 & 0.00065 \\
\hline Hydrochlorothiazide & 0.00049 & $1.646 \mathrm{E}-05$ \\
\hline
\end{tabular}

\section{A. FEA Simulation Results}

The following analytical models represent the pill holding plate, which is driven by the Z-Axis mechanism, experiencing the maximum load it may undergo from the heaviest pills $(1000 \mathrm{mg})$ filling all twelve cartridges. After physically testing with sample pills and a printed cartridge, it was determined that roughly 30 of these $1000 \mathrm{mg}$ pills would fill each cartridge completely. In order to do any simulated work on this plate, the maximum load was first calculated. Since the plate is given the correct material (PLA) in the simulation, with the corresponding material properties, the load from the weight of the plate is accounted for. Using a software called Cura, which is a tool used to create $3 \mathrm{D}$ printed models, the exact mass of each pill cartridge (47 grams) was found using the correct PLA plastic material. The maximum load from the twelve pill cartridges filled with thirty $1000 \mathrm{mg}$ pills each was found to be $9.064 \mathrm{~N}$. The total load was applied to the edges of each pill cartridge, as shown in purple in Fig. 11. The stress, strain, and displacement simulation for this loading case was completed using SolidWorks and is shown in Fig. 11-13.

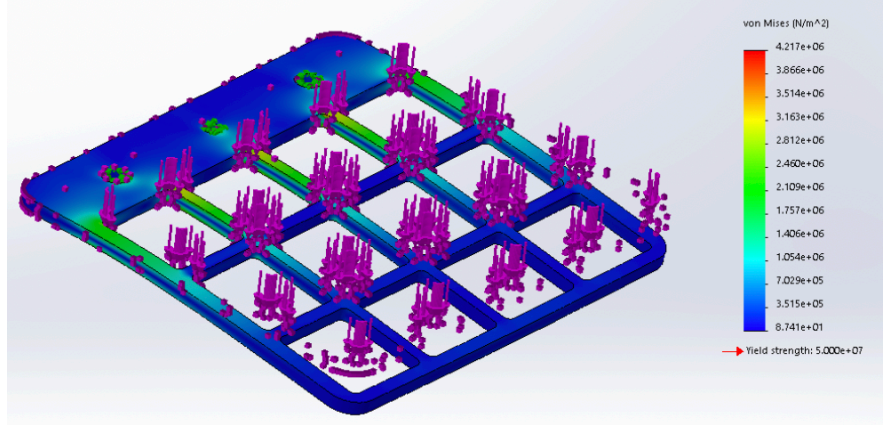

Figure 11. Stress Simulation

The purpose for the simulation conducted on the pill holding tray was to visualize and record the stress, strain, and displacement that the part would experience under maximum load. From these values, the factor of safety can be calculated, as well as the amount of deformation that will occur. Any part under a loading condition must be tested to see if failure will occur during operations.

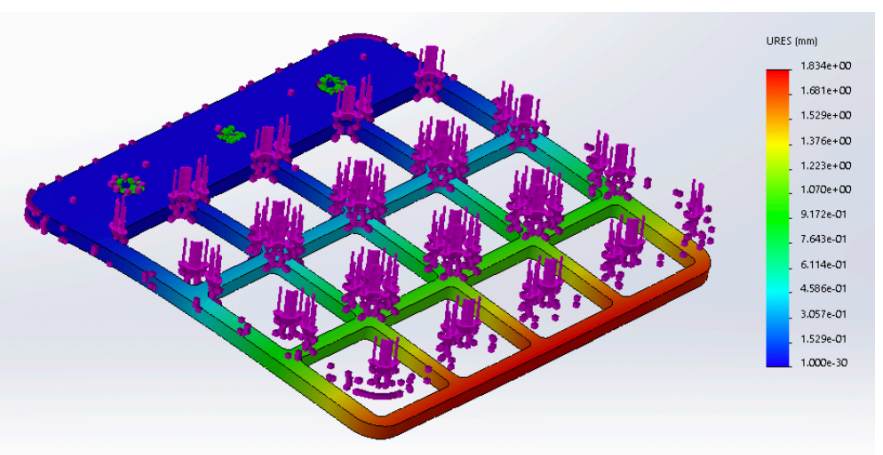

Figure 12. Displacement (Deformation) Simulation

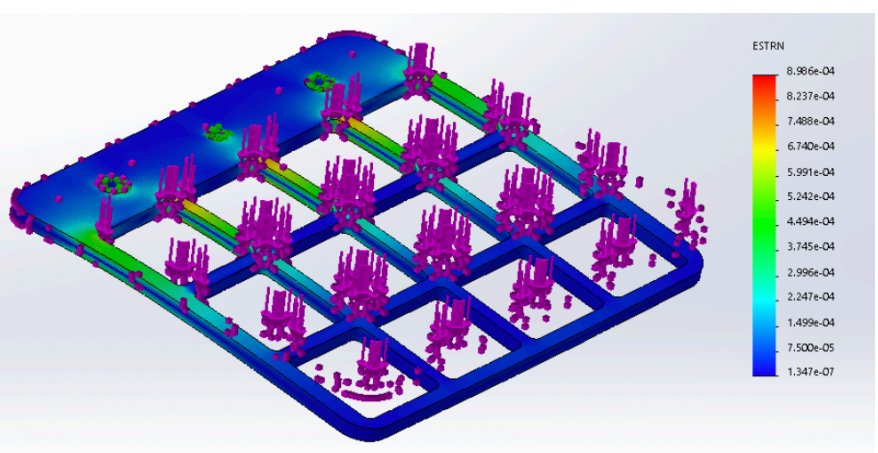

Figure 13. Strain Simulation

The factor of safety of the plate is represented by ' $n$ ', ' $\sigma_{M}$ ' (material strength), and ' $\sigma_{w}$ ' (working stress) as shown below. These simulations represent the maximum load that the pill holding tray may experience using the heaviest common pill (1000mg) to fill up each cartridge (30 pills). This tray for product $B$ has a thickness of .25inches and has a factor of safety of 11.9, in comparison to the product A which was .5 inches thick and had a factor of safety of $\mathbf{5 2}$.

$n=\sigma_{M} / \sigma_{w}$.

A high factor of safety is desired for this part, in case of any additional accidental weight when refilling the cartridges. Although the previous model had a higher factor of safety, it was deemed overkill and unnecessary, which led to the change in thickness. Modelling the plate as a 2D cantilevered beam, a shear-moment diagram was created from a free-body-diagram of the loading case. Here, the maximum load from the pills and cartridges and the load from the weight of the tray was needed, as well as the centroids of both forces. The fixed support was created at the center of the guide rail holes on the plate. The load from the weight of the plate was calculated to be roughly $1.628 \mathrm{~N}$ using Cura to find the mass (166grams). The centroids of the loads were found using the section properties tool in SolidWorks, with the maximum load of $9.064 \mathrm{~N}$ from the pills and cartridges and the weight load from the plate itself. 


\section{B. Analytical FEA Results}

In this section, an analytical approach has been implemented to calculate the applied load on each panel of the plate, modelling and identifying a panel that carries maximum load, conducting stress analysis, and finally obtaining the factor of safety of the plate against ultimate material strength.

Fig. 14 shows a free body diagram of load distribution through the plate structure that is produced from the twelve pill cartridges filled with thirty $1000 \mathrm{mg}$ pills and weight of the plate.

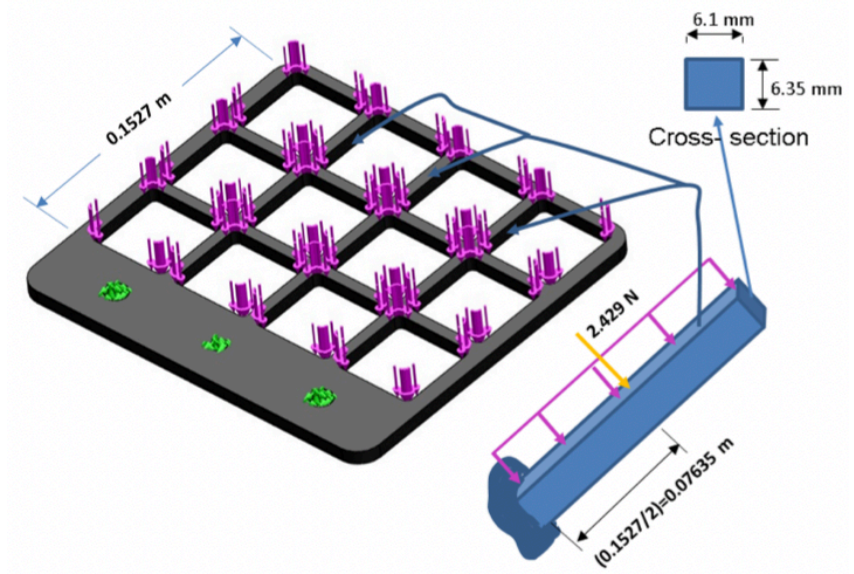

Figure 14. Load Distribution Free Body Diagram

Total load from the twelve pill cartridges, $P_{T}=9.064 \mathrm{~N}$

Load carried by two neighboring beam panel $=\frac{3(9.064)}{12}=$

$2.266 \mathrm{~N}$

Load carried by each exterior beam panel $=1.133 \mathrm{~N}$

Load carried by each middle beam panel $=2 * 1.133=$ $2.266 N$

Calculations above show that the middle beam carries the maximum load and hence maximum stress.

The load from the weight of the plate is $1.628 \mathrm{~N}$, and by using the beam panel volume $\left(0.1527 * 0.00635 * 0.0061 \mathrm{~m}^{3}\right)$ to the total volume, the weight for each beam panel is calculated as $0.163 \mathrm{~N}$. Hence, the total load (TL), as a result of both the twelve pill cartridges filled with thirty $1000 \mathrm{mg}$ pills plus weight of teach beam panel, for each middle beam panel can be calculated as

$$
T L=2.266+0.163=2.429 \mathrm{~N}
$$

Fig. 15, shows the load distribution through each middle panel that carries maximum total load of $2.429 \mathrm{~N}$. The maximum bending stress as a result of total load has been calculated based on the support cross section dimensions of the panel, and factor of safety has been obtained against ultimate material strength.

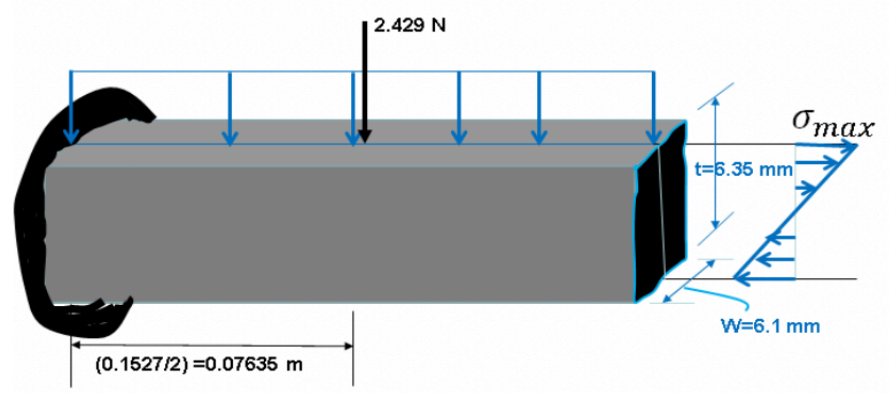

Figure 15. Bending Diagram

$C=\frac{6.35}{2}=3.175 \mathrm{~mm}$

$I=\frac{1}{12}\left(0.0061 * 0.00635^{3}\right)=1.302 \times 10^{-10} \mathrm{~m}^{4}$

$M_{\text {max }}=2.429(0.07635)=0.18545 \mathrm{~N}-\mathrm{m}$

$\sigma_{\text {max }}=\frac{M_{\text {max }} C^{-C}}{I}=\frac{0.18545 * 0.003175}{1.302 \times 10^{-10}}=4.5 \mathrm{MPa}$

F.S $=\frac{\sigma_{\text {Ultimate }}}{\sigma_{(\max ) \text { applied }}}=\frac{50 \times 10^{6}}{4.5 \times 10^{6}}=11.12$

$\%$ error $=\frac{\sigma_{\max \text { Analytical }}-\sigma_{\max \text { Simulated }}}{\sigma_{\max \text { Analytical }}}$

$\frac{4.5-4.2}{4.5} * 100 \%=6.66 \%$

Through SolidWorks FEA simulation, a maximum stress value of 4.2 MPa was found from the loading conditions, while the analytical maximum stress was found to be $4.5 \mathrm{MPa}$. Above calculations demonstrate that the maximum stress results lay within a reliable range (within a $6.6 \%$ variation) in comparison to FEA analysis. In addition, the same can be said for the factor of safety evaluation using both SolidWorks' FEA and analytical approach.

\section{DISCUSSION}

Preliminary trials of the two medicine dispenser designs were conducted comparing the behavior of the devices. The test done on the mechanisms shows that both machines A (PharmAid) and B (New model) are accurate and reliable. Through some modifications to the previous prototype, the team improved the dispensing time by altering the mechanism and the cartridge holder. The distributor for product A (Fig. 1) rotates about a principal axis using a stepper motor. The cartridges have a flat shape on the bottom. However, device B implements a narrow end on the cartridges, facilitating the suction system to obtain a pill more efficiently. Also, product B uses the X-Y-Z system for a faster response. The two apparatuses were under analysis to confirm that by making such modifications, the device would conduct its task more efficiently. Throughout these implementations, the team plans to create a quicker response while remaining efficient. A graph comparing time against the number of pills dispensed (Fig. 16) shows that product B has a faster response in dispensing medicine. The plot indicates that the time difference to administer one medication noticeably improved by a few seconds. The decision to change the distribution mechanism and the shape of the cartridge holder was correct. Product B shows a reduction in time of up to $21.34 \%$ compared to product A. Prototype A takes about six minutes and forty-three seconds to dispense twelve pills. On the 
other hand, product B takes about five minutes and one second to dispense twelve medicines. This product is intended to help people who require from eight to twelve different medications a day. The results prove that administering only one pill would not increase drastically; however, improving the time to dispense different medicines at once is a relevant development. Taking into consideration the new mechanism and the modification of the cartridges, one can agree that the results satisfy people's needs. Perhaps the rotation distributor took a few seconds longer to align the suction system with the cartridges making the system slower in operation.

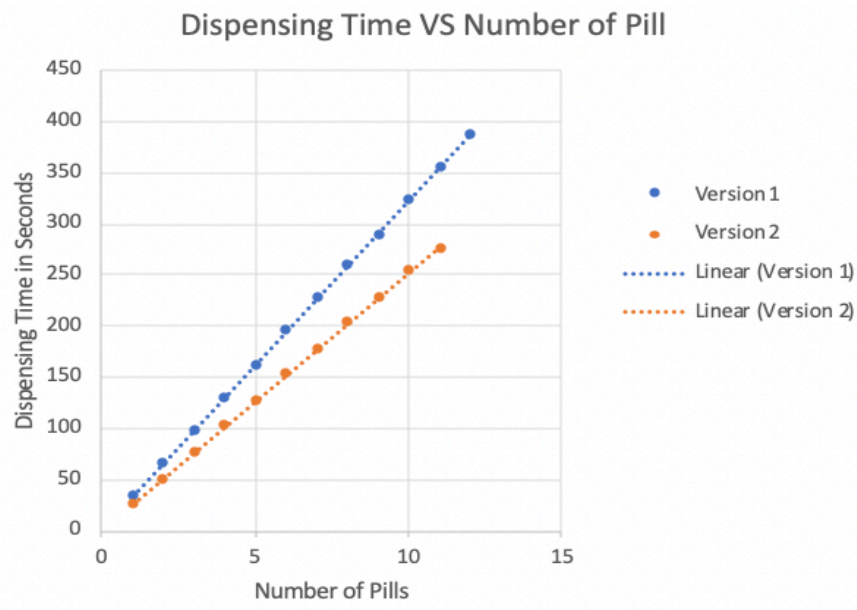

Figure 16. Time Response of Product A and B

Moreover, PharmAid deals with electronic components that create heat throughout the machine. Medicine is a delicate subject to work with, as heat can melt pills or capsules, leading to severe consequences. For prototype B, implementation of fans is necessary to prevent complications. A comparison diagram (Fig. 17) provides temperature disclosure of the two different models. Prototype A has a room temperature while in sleeping mode; however, the dispensing mode can bring the temperature up to 86 Fahrenheit, which can be a high enough temperature to melt a medicine. On the other hand, Prototype B has fans and a humidity sensor that controls the heat and detects the change that can alter electrical currents and temperature in the air. As shown in Fig. 17, product B reduces the dispensing mode temperature up to $10.47 \%$, creating a suitable environment for the average medicine.

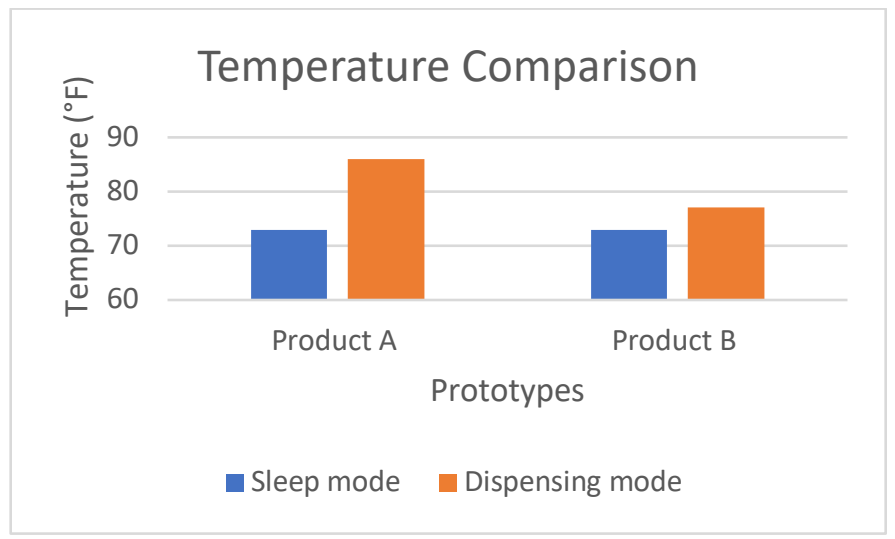

Figure 17. Temperature Comparison

\section{FUTURE WORK}

There are several aspects to consider in order to improve the project in the future, such as: noise reduction, smart phone application implementation, pill cartridge numbers, and liquid dispensing capabilities. Through several tests, the group found that the vacuum pump creates a relatively boisterous environment which can be reduced by creating a soundproof compartment in which to store the vacuum pump. Also, the implementation of a smartphone application will improve the effectiveness of the product. The creation of a smart phone app will make pill management operations even easier. In addition, the autonomous medicine dispenser is considered to have enough cartridges; however, adding two to four more cartridges will allow patients to manage more medicines. Furthermore, implementation of a liquid dispenser would be ideal for those individuals who have difficulty ingesting pill tablets and who would rather take liquid medications.

\section{CONCLUSIONS}

Throughout the analysis and the research done on PharmAid, the group agrees that the autonomous medicine dispenser is convenient and reliable. However, the team evaluated statistics on the product that reveal that the unit can be improved. Under the analysis, the temperature sensor, humidity sensor, vacuum compressor, ESP32, and the X-Y-Z distributor were improved in the project. The two different prototypes were compared to determine the best results for the user's convenience. In conclusion, the results satisfy the project goals. On the other hand, better applications can be added to increase the benefits for patients.

\section{ACKNOWLEDGMENT}

The authors would like to acknowledge their faculty advisors, Dr. Rahemi, Dr. Benalla, Dr. Bustamante, Dr. Elzawawy, Dr. Ducharme, and the Vaughn College Department of Engineering faculty for their technical support. Also, a special thank you to PETROELF S.A. for funding and supporting the project.

\section{REFERENCES}

[1] Hectic schedules cause most people to forget to take medicine: Study. (1998, Oct 05). Jet, 94, 27. Retrieved from https://search-proques com.vcproxy.vaughn.edu/docview/199988241?accountid=30591

[2] Wertheimer, A. I., and Santella, T. M., Medication compliance research: still so far to go. J. Appl. Res. Clin. Exp. Ther. 3 (3)254-261, 2003.

[3] Johnson, L. A. (2014, Apr 21). Smartphone apps remind patients to take their meds. The Epoch Times Retrieved from https://search-proquestcom.vcproxy.vaughn.edu/docview/1519533862? accountid=30591

[4] Santo, K., Chow, C. K., Thiagalingam, A., Rogers, K., Chalmers, J., \& Redfern, J. (2017). MEDication reminder APPs to improve medication adherence in coronary heart disease (MedApp-CHD) study: A randomised controlled trial protocol. BMJ Open, 7(10) doi:http://dx.doi.org.vcproxy.vaughn.edu/10.1136/bmjopen-2017-017540

[5] Algorithms; research from C. au and colleagues provide new insights into algorithms. (2011, May 12). Computer Weekly News Retrieved from https://search-proquest-

com.vcproxy.vaughn.edu/docview/864664469?accountid=30591 
[6] Dixit, Pooja. "Bresenham's Line Algorithm." LinkedIn SlideShare, 7 Feb. 2019, 130849319 .

[7] Shenzhen Yinghui Yuan Electronics Co., Ltd, Jimmy. "What Are the Advantages of Switching Power Supplies?" Shenzhen Yinghui Yuan Electronics Co., Ltd., 19 Jan. 2018, www.yhypower.com/industrynews/What-are-the-advantages-of-switching-power-supplies.html.

[8] Butler Winding. "Switch Mode Power Transformers FAQ." Butler Winding, Butler Winding, 2020, www.butlerwinding.com/switch-modepower-transformers-faq/.

[9] American Society of Health-System Pharmacists. ASHP guidelines: minimum standard for pharmacies in hospitals. Am J Health-Syst Pharm. 2013; 70:1619-30

[13]Schraven, M. H., Guarnieri, C., Baranski, M. A., Müller, D., \& Monti, A. (2019). Designing a development board for research on IoT applications in building automation systems. Waterloo: IAARC Publications.

doi:http://dx.doi.org.vcproxy.vaughn.edu/10.22260/ISARC2019/0012

[14] "The 10 Most Prescribed Medications for the Elderly and What They Do." UEW Healthcare, 6 Aug. 2019, www.uewhealth.com/10prescribed-medications-elderly/.

[15] J. Schmalz GmbH: Innovative Vacuum Technology from Schmalz. (n.d.). Retrieved from https://www.schmalz.com/en/vacuumknowledge/the-vacuum-system-and-its-components/system-designcalculation-example/theoretical-holding-force-of-a-suction-cup/
[10]ANSI. "Gaps Portal ." America Makes \& ANSI Additive Manufacturing Standardization Collaborative (AMSC), ANSI, 2019, www.ansi.org/standards activities/standards boards panels/amsc/Americ \{a-Makes-and-ANSI-AMSC-Overview.

[11]ISO. "Popular Standards." ISO, ISO, 3 Sept. 2019, www.iso.org/popularstandards.html.

[12] Brody, Jane E. "The Cost of Not Taking Your Medicine." The New York Times, The New York Times, 17 Apr. 2017, www.nytimes.com/2017/04/17/well/the-cost-of-not-taking-yourmedicine.html.

[16]Benjamin, Regina M. "Medication Adherence: Helping Patients Take Their Medicines as Directed." Public Health Reports (Washington, D.C. : 1974), Association of Schools of Public Health, 2012, www.ncbi.nlm.nih.gov/pmc/articles/PMC3234383/.

[17]Hydrocodone. (2019, April 25). Retrieved from https://www.ncbi.nlm.nih.gov/books/NBK548700/

[18]Simvastatin Dosage Guide with Precautions. (n.d.). Retrieved from https://www.drugs.com/dosage/simvastatin.html

[19]Center for Drug Evaluation and Research. (n.d.). Prilosec OTC (omeprazole) Information. Retrieved from https://www.fda.gov/aboutfda/center-drug-evaluation-and-research-cder/prilosec-otc-omeprazoleinformation 